\title{
Le thème de la frontière dans Le signe du Cancer de Mihail Sadoveanu
}

\section{Alexandra Vranceanu}

\section{(2) OpenEdition}

1 Journals

\section{Édition électronique}

URL : https://journals.openedition.org/cher/10310

DOI : $10.4000 /$ cher. 10310

ISSN : 2803-5992

\section{Éditeur}

Presses universitaires de Strasbourg

\section{Édition imprimée}

Date de publication : 1 décembre 2013

Pagination : 111-124

ISBN : 978-2-86820-560-5

ISSN : 1968-035X

\section{Référence électronique}

Alexandra Vranceanu, « Le thème de la frontière dans Le signe du Cancer de Mihail Sadoveanu », reCHERches [En ligne], 11 | 2013, mis en ligne le 08 février 2022, consulté le 09 février 2022. URL : http://journals.openedition.org/cher/10310 ; DOI : https://doi.org/10.4000/cher.10310

\section{(c) (i) (2)(2)}

Ce(tte) œuvre est mise à disposition selon les termes de la Licence Creative Commons Attribution Pas d'Utilisation Commerciale - Partage dans les Mêmes Conditions 4.0 International. 


\title{
Le thème de la frontière dans Le signe du Cancer de Mihail Sadoveanu
}

\author{
Alexandra Vranceanu \\ Université de Bucarest
}

Dendant la dernière moitié du $\mathrm{xIX}^{\mathrm{e}}$ siècle et la première moitié du P $\mathrm{xx}^{\mathrm{e}}$ siècle la littérature roumaine était fortement dominée par le modèle français. Cette forte influence qui se manifestait aussi dans d'autres domaines que la vie littéraire a généré un mouvement de refus des influences étrangères et de retour aux valeurs nationales, qui s'est concentré autour de la revue Semănătorul (Le Semeur) dirigée par Nicolae Iorga et dans la revue Viața românească (La Vie roumaine) dirigée par Garabet Ibraileanu (Durandin, 1979). Le conflit entre ces deux mouvements littéraires, la direction nationaliste et traditionaliste de N.Iorga et G. Ibrăileanu et la direction cosmopolite et européenne, dirigée par Eugen Lovinescu, domine l'espace littéraire roumain jusqu'à la fin de la deuxième guerre mondiale (Ornea, 1971: 125-169). Cette nouvelle querelle entre les «anciens» (les traditionalistes amoureux du folklore et des vieilles traditions roumaines) et les «modernes» (admirateurs de la culture cosmopolite de l'Europe) conduit à l'apparition de nombreux textes littéraires et scientifiques qui expriment l'angoisse identitaire des intellectuels roumains ${ }^{1}$. Ce clivage a

1 Sorin Alexandrescu pense qu' « en Roumanie il y a pratiquement deux cultures : le folklore et la culture des lettrés» (Alexandrescu 1999: 22) et que le prestige des traditions fait ainsi que le folklore roumain peut être considéré comme "le vrai classicisme roumain" (Alexandrescu 1999: 24). L'histoire littéraire roumaine se construit au XIX ${ }^{e}$ siècle, et porte l'influence du courant romantique et de la vision de Herder, ce qui explique le rôle central occupé par le folklore et par les traditions. Mais le folklore reste une importante source d'inspiration et un réservoir de mythes pour les écrivains roumains du $\mathrm{Xx}^{\mathrm{e}}$ siècle aussi, quand, à côté des écrivains d'avant-garde comme Tristan Tzara, Ion Vinea, Gherasim Luca, il y a des écrivains qui décrivent la vie du village et ses traditions. 
semblé si profond aux historiens littéraires, qu'ils ont séparé et étiqueté les écrivains selon leurs préférences, soit pour une direction, soit pour l'autre (Călinescu, 1983). Mais souvent la lecture des textes canoniques nous réserve des surprises lorsqu'on observe que les écrivains considèrés traditionalistes, comme par exemple Mihail Sadoveanu (1880-1961), réfléchissent aussi à la culture européenne et que la description des traditions ne conduit pas nécessairement au nationalisme. Un exemple intéressant est constitué par le roman Le signe du Cancer publié en 1929 par Mihail Sadoveanu, dans lequel je me propose d'analyser le thème de la frontière, de l'étranger, de l'ailleurs.

\section{Les limitations du canon littéraire}

Lors de sa longue carrière de plus de 50 ans, Mihail Sadoveanu a souvent décrit la Moldavie: dans des romans historiques comme Les Frères Jder, Le signe du Cancer ou Nicoară Fer à Cheval, dans des romans qui prennent comme sujet la vie des paysans (Le hachereau, L'auberge d'Ancoutza), et la vie des petites villes de province (Haia Sanis, L'Endroit où il ne s'est rien passé) ou dans ses récits de chasse et de pêche, par exemple Țara de dincolo de negură (Le pays au-delà du brouillard) ou Împărăția apelor (L'Empire des eaux). Sadoveanu débute en 1904 avec quatre volumes (dont trois récits et un roman), et sa productivité légendaire l'amène directement au centre du canon littéraire. Il fait partie de l'Académie roumaine dès l'âge de 23 ans, il reçoit de nombreux prix avant la deuxième guerre mondiale. Après 1944 il illustre le réalisme socialiste dans des romans très appréciés par le régime communiste, comme par exemple Nicoara Potcoavă et Mitrea Cocor ${ }^{2}$. Malheureusement, la littérature de Mihail Sadoveanu a été la victime du canon littéraire communiste. Son œuvre a été surinterprétée dans les manuels, qui lui ont associé une connotation nationaliste, en le présentant comme un rhapsode d'une Moldavie idéale à la nature extraordinaire, l'histoire pleine de héros nationaux et aux personnages spécifiques, des paysans moldaves qui représentent bien la Roumanie profonde (Ciopraga, 2006). Son œuvre a bénéficié de nombreuses éditions et les critiques lui ont dédié des monographies importantes (Manolescu, 1976 et Spiridon, 1982). Et pourtant l'œuvre de Sadoveanu reste associée aux mouvements nationalistes et anti-européens. Sadoveanu semble d'ailleurs le candidat idéal pour une

2 M. Sadoveanu entre dans la politique et devient membre du Parlement communiste (Marea adunare nationala). A ce sujet voir Durandin, 1994, Alexandrescu, 1990 et Dragomir, 2003. 
histoire littéraire inspirée par Herder, car son œuvre prend comme thème la vie des paysans, décrit une nature non-contaminée par la civilisation, s'inspire de l'histoire nationale et de ses mythes. Sa présence constante dans le canon littéraire a conduit donc à des conclusions comme: "Sadoveanu conçoit son œuvre comme un édifice ayant la dimension du pays tout entier» (Ciopraga, 2006: 144), "dans sa structure intérieure, l'œuvre de Sadoveanu est la création d'un rhapsode qui possède une connaissance exceptionnelle de la terre et des hommes» (Ciopraga, 2006: 144), et «nous aimons nous reconnaittre en tant que peuple dans de pareilles œuvres...» (Ciopraga, 2006: 144).

Monica Spiridon observe que:

Dans des manuels roumains d'histoire littéraire, une formule comme «Sadoveanu, l'écrivain archéologue del'espace national » est un des stéréotypes les plus résistants. Tombant droit dans le piège de la vraisemblance quasiment scientifique des topographies romanesques construites par Sadoveanu, l'école finit par institutionnaliser le statut de musée géographique, ethnologique, historique de ses fictions. (Spiridon 2004: 28, Manolescu 1976: 180-211)

Du point de vue de la technique littéraire, les récits de Mihail Sadoveanu sont réalistes avec des traits romantiques, mais il ne s'intègre pas clairement dans un mouvement, même si ses débuts se placent sous le signe de la revue Semănătorul (Le Semeur) dirigée par Nicolae Iorga, qui a généré le "Semănătorism », courant littéraire nationaliste et traditionaliste. Sadoveanu publie ensuite dans la revue Viaţa românească (La vie roumaine) de G. Ibraileanu, centre du courant littéraire nommé Poporanism (populiste), mais sa poétique ne peut être réduite aux idées discutées dans le cadre de ces mouvements littéraires (Ornea 1971). Il serait plus facile de classer la technique narrative de Mihail Sadoveanu à l'aide de catégories négatives: ses romans et récits ne sont pas modernistes, il ne suit pas les mouvements d'Avant-garde, ni l'exemple de Proust ou Gide, qui fascinait les écrivains roumains de l'époque. Il publie ses romans et récits dans le contexte de l'explosion moderniste des années 1920 et 1930 et ils sont perçus comme archaïques. Il est souvent étiqueté par rapport à sa distance des modèles préférés par ses contemporains, qui avaient découvert avec grand enthousiasme le modernisme à travers, le plus souvent, l'influence française. Et pourtant, Sadoveanu met en scène de manière très moderne la rencontre entre l'Occident et l'Orient dans Le signe du Cancer. 


\section{Une Moldavie «lointaine»}

En matière de technique littéraire Sadoveanu conserve le modèle narratif du XIX ${ }^{\mathrm{e}}$ siècle et l'amour pour les traditions apparaît aussi comme thème dans ses romans. Même lorsque l'intrigue a lieu dans la Moldavie contemporaine, comme par exemple dans Le hachereau, Sadoveanu décrit un espace traditionnel menacé par la modernité, un espace qui semble mythique et atemporel. La Moldavie s'invente petit à petit dans la description de son passé historique et à travers la description détaillée de la vie des habitants des villages et des petites villes de province. Plusieurs raisons expliquent l'impression que Sadoveanu décrit une Moldavie lointaine et mythique.

La première est le refus du modernisme comme formule littéraire.

La deuxième raison se retrouve dans la mise en scène du conflit entre tradition et modernité par ses textes: les personnages de Sadoveanu ne semblent pas préparés à accepter la vie moderne, et le conflit entre les changements produits par la vie moderne et la vie patriarcale apparaît souvent $^{3}$. Dans tous ses romans et récits, Sadoveanu fait l'éloge de la vie traditionnelle au sein de la nature, et la nature est souvent représentée comme un paradis menacé par la modernisation. Dans ses récits de chasse la description de la nature souligne le conflit entre la modernité, représenté par l'habitant de la grande ville, en général le narrateur, et les êtres primitifs qui savent communiquer avec la nature, sans la dominer ou la détruire. Cette approche romantique se retrouve dans plusieurs textes, comme Le signe du Cancer, Țara de dincolo de negură [Le pays au-delà des brumes] ou Le Hachereau. L'extraordinaire constance de la technique littéraire et du style de Sadoveanu, nous permet de reconnaître cette constante dans les romans historiques, les récits de chasse et de pêche et dans les romans de la vie de province.

La troisième raison qui donne l'impression que Sadoveanu décrit une Moldavie éloignée, dans le temps et l'espace, est le rôle central qu'il donne au mythe et à l'histoire. Même lorsqu'il décrit une intrigue vraisemblable,

3 Par exemple, dans Le Hachereau (Baltagul), Vitoria Lipan, une paysanne qui a passé sa vie dans un village de montagne isolé fait un long voyage pour retrouver son mari, un riche berger. Quand elle comprend qu'il avait été tué, elle met en scène une sorte de procès judiciaire qui lui permet, avec les moyens simples de la vie traditionnelle au village, de découvrir les coupables et de réinstaurer l'ordre. Elle fait semblant de demander l'aide aux représentants de la civilisation moderne (la police, la préfecture, le maire etc.), mais elle réussira à venger son mari sans s'en servir, grâce à sa connaissance de la vie patriarcale et aux habitudes des paysans. 
inspirée par des sources historiques, Sadoveanu laisse l'impression qu'il récupère un temps légendaire et éloigné.

Ces raisons peuvent être des raisons superficielles pour considérer Sadoveanu un écrivain préoccupé exclusivement par l'esprit national et, à une lecture plus attentive, on peut observer que le rapport entre la culture roumaine et l'Europe ne le laissait pas indiffèrent. Dans son roman publié en 1929, Le signe du Cancer (Zodia Cancerului sau vremea Ducăi vodă), Sadoveanu décrit l'histoire de la Moldavie à travers les yeux d'un Français, utilisé comme personnage "réflecteur», dans le sens donné à ce terme par Henry James. Il ne s'agit pas ici de mettre en relation l'écriture de Sadoveanu avec les techniques littéraires car l'écrivain ne cherche pas à innover de ce point de vue. Le roman Le signe du Cancer paraît au moment où les romanciers roumains faisaient l'expérience du roman à la première personne, de l'«authenticité» sous l'influence de Gide ou de Proust. Sadoveanu ne reçoit pas ces influences au niveau de la technique romanesque. Il emploie dans Le signe du Cancer le personnage réflecteur qui ne comprend pas, qui ne sait pas comment interpréter ce qu'il voit. C'est à travers les yeux de l'étranger, l'abbé de Marenne, que se construit l'image de la Moldavie du $\mathrm{XVII}^{\mathrm{e}}$ siècle et il ne s'agit pas d'une image idéalisée, car aucun héros national n'apparaît, ni aucune action mémorable. La technique du manuscrit trouvé à la Bibliothèque nationale française permet à Sadoveanu de développer le thème de la frontière, de l'éloignement, de l'ailleurs car, dans son voyage vers «le grand Turc», l'abbé se sent au-delà du monde civilisé. L'image de la Moldavie qui se forme grâce au regard ébloui de l'abbé de Marenne dans ce roman ambigu n'a rien d'une rhapsodie où d'une épopée nationaliste.

\section{Le voyage Vers le grand Turc: la reconstruction de la Moldavie à travers les yeux de l'Autre}

Le signe $d u$ Cancer est un roman historique, un roman de voyage et un roman d'amour. Je m'arrêterai ici seulement sur le thème du voyage et sur la reconstruction de l'histoire à travers le regard de l'étranger. L'action du roman a lieu au XVII ${ }^{\mathrm{e}}$ siècle, lorsque l'abbé Paul de Marenne traverse la Moldavie pour arriver dans l'Empire Ottoman. L'abbé de Marenne, est le messager et l'ambassadeur de Louis XIV, mais il voyage incognito, et prétend qu'il a des buts scientifiques. Il rencontre le prince moldave Alecu Ruset, qui lui sert de guide en Moldavie et avec qui il échange des propos concernant la politique des Moldaves. Le lecteur comprendra que la mission de l'abbé de Marenne auprès du Sultan est d'encourager les Turcs dans leurs luttes contre 
l'Empire allemand de Léopold. À l'époque, la Moldavie était autonome, mais non indépendante car elle était la vassale des Ottomans, auxquels elle payait un tribut toujours plus élevé. La fonction de prince régnant ne s'obtenait plus comme au siècle précèdent, où les seigneurs moldaves choisissaient parmi eux mais le Sultan faisait la nomination. Cette fonction s'achetait avec de fortes sommes d'argent, qui étaient ensuite récupérées par une politique fiscale paupérisante. Sadoveanu ne choisit donc pas une époque de gloire, mais une période de décadence de la Moldavie, très différente de la France de Louis XIV, d'où vient l'abbé de Marenne. Ce contraste profond ne reste pas sans effet sur la signification profonde du roman.

En utilisant le stratagème narratif de l'étranger, mais sans renoncer totalement au narrateur omniscient qui éclaire de temps en temps certains détails, Sadoveanu réussit à produire un récit convaincant. Le narrateur affirme que son récit reprend un document de voyage écrit par l'abbé:

La relation manuscrite de monsieur Paul de Marenne, abbé de Juvigny, qui se trouve à la Bibliothèque nationale de Paris, abonde en considérations morales et philosophiques sur ce voyage intitulé Vers le Grand Turc. (Sadoveanu 1981: 48)

Ce stratagème narratif permet à Sadoveanu de présenter les pensées secrètes de l'abbé et de compléter sa vision par la voix d'un narrateur plus compétent:

Homme de son temps, le narrateur ne pouvait manquer de mêler à ses dires tout ce qui pouvait servir son propre intérêt, glorifiant l'époque du Roi Soleil et vantant les mérites de son protecteur, monsieur Charles Colbert marquis de Croissy, ministre des Affaires étrangères et frère du grand Colbert. Mais tout cela nous intéresse moins. Nous préférons suivre notre voyageur dans des détails qui nous surprennent aujourd'hui et qui, en son temps, pouvaient sembler risibles. (Sadoveanu 1981: 48)

Le regard de l'abbé de Marenne n'échappe pas aux stéréotypes de l'écriture exotique, que Sadoveanu emploie pour donner une substance vraisemblable au récit de l'étranger. L'abbé voyage dans une Moldavie détruite par les nombreuses guerres, par les incursions des Tartares, par le manque d'ordre et organisation et, surtout, par la corruption. Il rencontre dans son passage des maisons pauvres, des moulins brûlés, il observe que les gens préfèrent passer par la forêt que par les rues principales, et que les ponts de pierre manquent. Dans ce contexte, les paysans de la Moldavie sont décrits, en respectant le contrat narratif du personnage réflecteur avec les stéréotypes de l'exotisme, comme des troglodytes ou des sauvages. D'une part, Marenne reprend le mythe du noble sauvage «Plus je pousse vers 
l'Orient, plus je constate que les hommes sont proches ici de la nature et de Dieu » (Sadoveanu, 1981, 27), d'autre part il constate le caractère primitif de ces paysans: «L'Hôte étranger contempla une fois encore les êtres primitifs de ces lieux, avec la plus vive curiosité, puis il demeura silencieux [...]» (Sadoveanu, 1981, 55) ou «l'apparition grotesque du sauvage» (Sadoveanu 1981: 48).

Le regard posé par Marenne sur les réalités de la Moldavie du XVII siècle oscille entre stupéfaction et incompréhension. Lorsqu'il demande au prince Ruset de lui montrer de belles constructions, par exemple des ponts de pierre, la réponse du Moldave lui semble ironique, car Ruset fait l'apologie des «huttes et des grottes que Dieu le père octroie avec largesse à ses créatures» (Sadoveanu 1981: 43). Marenne n'est pas sur que le prince Ruset ne plaisante pas et il a le même doute lorsque, devant l'anarchie qui règne en Moldavie, celui-ci fait l'apologie de l'ordre: «'La justice et l'ordre sont les premiers éléments d'un Etat', objecta l'abbé de Marenne, piqué au vif par l'indifférence de son compagnon. 'Cela est bien possible, Monsieur l'abbé, mais voilà des bienfaits dont Dieu nous réserve la surprise pour quelque siècle à venir'» (Sadoveanu 1981: 46), lui répond le prince moldave.

Le contraste entre la civilisation française et le monde sauvage des Moldaves apparaît aussi dans l'épisode du festin chez Lazarel Griga, un petit propriétaire terrien que les voyageurs rencontrent par hasard dans la forêt et qui les invite chez lui. À la fin du repas, bien arrosé de bon vin, la femme de Lazarel Griga offre aux invités une soupe spéciale, en roumain potroc, qui aide à éviter les effets négatifs de l'alcool. L'abbé demande la recette, mais il ne réussit pas à la comprendre, ce qui attire la remarque de Ruset: «Vous ne rencontrerez nulle part, dans le monde civilisé, Monsieur de Marenne, pareille manière de préparer ce bouillon» (Sadoveanu 1981: 39). Le plus souvent c'est Ruset qui souligne les contrastes entre le caractère sauvage de son pays et la civilisation (occidentale).

Si l'abbé note l'absence des signes de la civilisation (occidentale, mais jamais nommée), il note aussi la grande beauté de la nature, et ce rapport apparaît souvent dans les textes qui décrivent des voyages «exotiques». Marenne est fasciné par le spectacle de la forêt moldave, mais son enthousiasme rencontre l'indifférence des Moldaves, qui sont trop proches de la nature pour l'apercevoir. Dans une clairière l'abbé de Marenne, le prince Ruset et leurs guides rencontrent les urus (aurochs), dont le symbole héraldique représente le pays moldave. Le Français est très impressionné par la beauté de la scène: 
L'abbé fut pénétré de piété au spectacle fugitif du miracle de la divinité, fascinant et non pareil au fil de l'éternité. Il sentit le besoin d'interroger, de se laisser aller à des envolées lyriques; mais ses compagnons suivaient leur chemin, indifférents. Il éperonna à son tour le cheval, avec, de nouveau, un sentiment de dépit. (Sadoveanu 1981: 63)

Les impressions de l'abbé caractérisent la vision de l'homme civilisé qui contemple la beauté naturelle, invisible pour le primitif. Cette différence de vision apparaît souvent chez Sadoveanu dans les récits de chasse, où l'esthète est un citadin fier de son équipement mais incapable de réussir à chasser le moindre animal. L'esthète revient de l'expédition fasciné par la beauté de la nature, mais humilié par son compagnon de chasse, le paysan qui, sans admirer le paysage, réussit à dominer la nature avec des moyens plus simples, mais plus efficaces. Dans Le signe du Cancer Sadoveanu attribue la vision du «civilisé» au Français, pour souligner le contraste entre l'Occidental et les Moldaves. Le rapport est décrit sans ironie, et les habitants des deux mondes réussissent à lier des relations d'amitié. Ce type de rapport apparaît aussi dans l'exotisme, mais Sadoveanu le réinterprète en décrivant son propre pays comme s'il s'agissait d'un espace «lointain » et "étranger».

Parmi les stéréotypes de l'écriture exotique, le thème du paradis terrestre occupe une place importante. Marenne tend à idéaliser la Moldavie, mais le prince Ruset corrige ses impressions, ce qui montre que les principes de la civilisation occidentale ne lui manquent pas. Lorsque le Moldave observe que son pays est mal placé («Nous vivons des temps difficiles et je me demande pourquoi Dieu a cru devoir nous placer aussi mal»), l'abbé français lui répond: "Je ne trouve pas, prince, Dieu a créé ici un Éden» (Sadoveanu 1981: 49). Dans son pays Ruset voit la crise politique plutôt que la beauté édénique et il complète ainsi l'observation de l'abbé: "Vous dites vrai : un Éden dévasté » (Sadoveanu 181:49).

\section{La frontière entre empires, cultures, civilisations}

Une idée centrale du Signe du Cancer est que la Moldavie constitue une frontière entre Orient et Occident, ce qui conduit tantôt à une synthèse, tantôt à un conflit. Lorsque l'abbé rencontre pour la première fois le prince moldave Ruset, il observe au début les marques orientales de sa tenue:

Il chevauchait, selle haute, étriers courts, à la manière des impies ismaélites, ce qui, sur le moment, n'eut pas l'heur de plaire à monsieur l'abbé de Marenne. (Sadoveanu 1981: 29) 
Mais quand Ruset le salue en français, Marenne change son impression: «Il salua en un assez bon français et s'inclina devant l'étranger" (Sadoveanu 1981: 29). Cette synthèse entre Orient et Occident se retrouve aussi dans la description faite par Ruset à sa formation:

[...] j'ai eu le plaisir et le bonheur de voir le pays de Votre Seigneurie, de faire des études en Pologne, de vivre aussi quelques temps à Istanbul, parmi les descendants de Byzance. (Sadoveanu 1981: 50)

Marenne considère le prince Ruset plus proche de la culture occidentale que de la culture orientale: "Vous êtes plutôt des nôtres que de l'Orient" (Sadoveanu 1957: 78, ma trad.), dit le Français au Moldave.

L'amitié profonde qui naît entre l'abbé français et le prince moldave fait demande que Marenne lui demandent les causes qui expliquent l'insécurité et l'extrême pauvreté des gens. L'explication de Ruset fait référence au conflit entre l'Orient et l'Occident: selon lui, le méchant sort des Moldaves est dû au fait que leur pays se trouve à la frontière entre l'Orient et l'Occident. L'Occident est représenté par la France, un pays bien organisé («rânduit» Sadoveanu 1957: 33) et heureux. L'Orient est représenté par les riches empires de l'Inde et des Ottomans. La Moldavie est la frontière qui sépare ces mondes et le théâtre de guerre de ces puissants empires:

[...] ici, entre ces mondes bienheureux, Dieu a jeté des bornes. Les empereurs au service de Belzebuth et les empereurs au service de la Croix ont choisi ces lieux pour porter leurs guerres. Ceci étant, le pays déchoit et se dépeuple. (Sadoveanu, 1981: 50) ${ }^{4}$

Le concept de frontière apparaît aussi dans une discussion menée par l'abbé de Marenne avec le représentant de l'église catholique, Pater Guido, un vieil Italien qui habite à Iași depuis longtemps. Il présente la Moldavie comme la frontière du monde des barbares, «hotarul lumii barbarilor» (Sadoveanu 1957: 103).

La synthèse entre les cultures de l'Orient et de l'Occident se manifeste aussi au niveau des rapports diplomatiques. Les cérémonies des Moldaves ne sont pas primitives et l'abbé est frappé par la cuisine raffinée et par la qualité du vin. Il est reçu à la cour de Iași par un discours en latin fait selon les règles connues de la rhétorique classique, ce qui le surprit aussi. De Marenne est encore plus choqué par la subtilité des intrigues byzantines des princes moldaves, qui mangent ensemble même s'ils se détestent et par leur

4 «Aicea însă, între lumi fericite, Domnul Dumnezeu a însemnat cu degetul hotar. Împărații lui Belzebut și împărații Mielului lumii aici au ales loc al războiului lor. Fiind așa, țara cade și se pustiește de oameni» (Sadoveanu 1957: 33). 
discours machiavéliques. Aux demandes plus précises de l'abbé concernant les conflits latents et incompréhensibles entre les princes moldaves, Alecu Ruset cite la tradition byzantine et affirme qu'il se défend de ses ennemis avec des armes indirectes, en réseaux superposées, selon les méthodes de se ancêtres de Byzance «în rețele suprapuse după metoda strămoșilor noștri de la Bizanț» (Sadoveanu 1957: 83). Byzance est dans ce roman de Sadoveanu un mythe déchu. La grande culture byzantine est réduite à ce qu'elle était devenue au XviI ${ }^{\mathrm{e}}$ siècle, quand les Grecs du quartier du Phanar avaient trouvé un mode de vie en s'intégrant dans le complexe appareil administratif et politique des Ottomans. De l'ancienne grandeur, il ne lui reste que la rhétorique compliquée, la corruption et les intrigues.

Ce contraste entre le caractère primitif de la Moldavie et son côté raffiné est présent dans la description de la capitale de la Moldavie, Iași, qui est décrite comme un espace chaotique, développé trop vite et sans aucun ordre, une ville orientale: «rămăsese un oraş al Orientului » (Sadoveanu 1957: 84). Par contre, arrivé à Constantinople, l'abbé voit dans l'ancienne capitale de Byzance son passé chrétien:

Monsieur de Marenne soupira en songeant que Dieu, pour châtier ses enfants bien-aimées, avait cru bon de permettre aux impies d'asseoir pour un temps leur camp dans le plus beau jardin du monde, en ce sérail enchanté que la mer classique vient baigner et envelopper de son doux murmure. (Sadoveanu 1981: 327).

Il y a deux observations synthétiques sur les Moldaves que le narrateur du roman attribue à l'abbé français. La première concerne le caractère primitif des contrées sauvages:

Ces lieux curieux étaient marqués par une telle solitude désolée que ses amis français n'auraient jamais pu s'imaginer, quelle que fut leur imagination; tant il est vrai que l'on trouve parfois, aux antipodes du monde civilisé, de ces choses restées intactes depuis les débuts de la Création ayant conservé toute leur mystérieuse beauté. (Sadoveanu 1981: 223)

Les contrastes du paysage lui semblent s'harmoniser avec «le tempérament de ce peuple primitif» (Sadoveanu 1981: 223). La deuxième réflexion concerne la civilisation corrompue, héritière des Byzantins, rencontrée à la cour moldave:

La manière de faire de ces disciples de Byzance était sans doute moins directe et moins brutale; mais elle ne laissait pas d'être pleine de fiel et perfide, puisqu'elle ambitionnait de s'assimiler à Dieu et au Destin. (Sadoveanu 1981: 173) 
L'image de la Moldavie se construit dans les mémoires de l'abbé entre ces deux caractéristiques contrastives: la primitivité et la perfidie byzantine.

L'histoire d'amour entre le prince Ruset et Catrina, la fille du prince régnant de la Moldavie Duca vodă, son ennemi le plus grand, se développe sur ce fonds de perfidie byzantine - l'abbé suspecte même Ruset de vouloir séduire la princesse Catrina pour accéder au pouvoir, mais ce doute ne l'empêche pas de sauver la vie de son ami lorsque le père de Catrina le menace. Le grand mérite de ce roman est que ses narrateurs n'exaltent les mérites d'aucune nation et ne les critiquent pas non plus; la rencontre entre les personnages appartenant à plusieurs cultures se fait, paradoxalement, sous le signe de l'amitié et de la trahison. Du point de vue de la politique, il y a des différences importantes. La mission de l'abbé de Marenne ne tient pas compte des intérêts de la Moldavie car Louis XIV soutient les Ottomans dans leur lutte contre les Allemands, ce qui n'échappe pas au prince Ruset. Malgré son amitié pour Marenne, Ruset confirme devant Duca voda qu'il est un ambassadeur de Louis XIV, en trahissant le secret de son ami. L'ambivalence des points de vue sur la politique des personnages du roman est contrebalancée par la facilité avec laquelle ils sont toujours prêts à donner la priorité à leurs relations d'amitié ou d'amour.

\section{Pourquoi Sadoveanu choisit-il comme personnage réflecteur un Français?}

À l'époque où Sadoveanu publie Le signe du Cancer, la scène littéraire roumaine est dominée par la littérature française. La fascination des Roumains pour la France était si puissante que certains écrivains, comme Benjamin Fondane ou Eugène Ionesco partent à Paris rejoindre le centre qui dominait depuis longtemps la littérature roumaine (Cornis Pop 1996: 114 et Spiridon 2004: 166-168). Le choix de Sadoveanu prenant un Français pour témoin du monde occidental peut s'expliquer ainsi.

Les écrivains et les historiens roumains utilisaient par ailleurs le français comme langue de communication vers le monde "civilisé», l'Europe occidentale, devant laquelle ils essayaient de présenter leur culture. Le fait que les milieux académiques parisiens étaient durant la première moitié $\mathrm{du} \mathrm{xx}^{\mathrm{e}}$ siècle les destinataires du discours de légitimation porté par les historiens roumains a aussi une explication d'ordre politique: la France avait joué un rôle central dans le procès d'unification de la Moldavie et de la Valachie au XIX ${ }^{e}$ siècle et elle était restée, pour les Roumains, le centre absolu de l'Europe. On s'explique ainsi les éléments communs entre la fiction de 
Sadoveanu et l'activité de Nicolae Iorga, historien roumain qui avait obtenu son doctorat à la Sorbonne et qui y donnait des cours sur la culture de l'Europe orientale. Nicolae Iorga, collaborateur de Sadoveanu à la revue Semănătorul [Le Semeur], publie à Paris en 1922 un volume intitulé Formes byzantines et réalités balkaniques, et en 1923 un autre, Relations entre Orient et l'Occident, tous deux inspirés par ses cours donnés à la Sorbonne. En 1935 il publie Byzance après Byzance. Continuation de l'histoire de la vie byzantine auprès des presses de l'Institut des Études Sud-Est Européennes, qu'il avait fondé à Bucarest comme preuve de son intérêt pour les recherches sur la contribution de l'Europe orientale à la pensée moderne. Iorga essaye de montrer la continuité entre une grande culture, synthèse du monde latin et grec, et l'Europe orientale. Ses contributions scientifiques sont une preuve de l'intérêt que portaient les intellectuels roumains, dans les années 1920 et 1930, à la légitimation de la culture roumaine devant les Français, vus comme symbole du monde occidental. Le roman de Sadoveanu s'inscrit dans cette tendance.

Lors d'une fête organisée par Duca vodă pour l'abbé de Marenne, le Français fait la connaissance de Miron Costin, un personnage qui a vécu au $\mathrm{XVII}^{\mathrm{e}}$ siècle et a écrit une histoire de la Moldavie ${ }^{5}$. L'humaniste lui parle de l'origine romane des moldaves et commence par un éloge pour leur passé: «Et pourtant ce pauvre pays a eu jadis des fils qui ne lui ont pas fait honte, réellement de dignes descendants de nos ancêtres du temps de l'Empire de Rome» (Sadoveanu 1981: 172) ${ }^{6}$. Devant la surprise de l'abbé, Costin pérore:

Et soudain ce boyard de fière allure, s'échauffant, se lança dans mille considérations historiques, scientifiques et théoriques, aux fins de faire-valoir les témoignages à même de vaincre les doutes du noble Français. À vrai dire, Marenne avait plus que des doutes: il n'ajoutait aucune foi à ce qu'on lui racontait. Rien, en effet, ne pouvait rappeler en ces confins barbares la grandeur des fils de la louve. "Manie de savant et contes de dormir debout», songea-t-il, en prêtant une oreille distraite aux propos de son compagnon. Le temps, il est vrai, a démontré que le savant logothète avait raison; mais l'abbé avait plus raison encore, puisque la chose le laissait indiffèrent. (Sadoveanu 1981: 172)

L'indifférence de l'abbé devant le discours où Miron Costin explique la continuité entre la culture romaine et le monde moldave exprime la vision

5 Par ce jeu narratif, Sadoveanu fait une substitution significative: ce sont les chroniques de Miron Costin (et de Ion Neculce) qui ont inspiré Le signe du cancer, mais il les remplace par les chroniques fictives de l'abbé de Marenne intitulées Vers le Grand Turc.

6 Voir à ce sujet Simion 2001. 
de Sadoveanu, sans illusion sur les clivages de l'Europe. En choisissant un personnage qui vient du temps de Louis XIV, un moment de gloire de la culture française et en le mettant en parallèle avec la Moldavie contemporaine, Sadoveanu renforce le thème de la frontière, qui donne le sens de son roman.

\section{Bibliographie}

Alexandrescu, S, 1990, «Une culture moderne de l'interstice. La littérature roumaine d'après-guerre", in Les Temps modernes, janv., p. 136-158.

Alexandrescu, S., 1999, La modernité à l'Est. 13 aperçus sur la littérature roumaine, Pitesti, Editura Paralela 45.

Călinescu, G., 1982, Istoria literaturii române de la origini până în prezent, București, Editura Meridiane, p. 615-630.

Ciopraga, C., 2006, «Une vision roumaine de l'existence: Sadoveanu », in Synergies, 2006, vol. 1, p. 139-144.

Cornis Pop, M., 1996, The Unfinished battles. Romanian Postmodernism Before and After 1989, Iași, Editura Polirom.

Crohmălniceanu, O.S., 1972, Literatura română între cele două războaie mondiale, vol.I., București, Editura Minerva.

Dragomir, L., 2003, «L'implantation du réalisme socialiste en Roumanie», in Sociétés et Representations, $\mathrm{n}^{\circ} 1,15, \mathrm{p} .307-324$.

Durandin, C., 1979, «Les intellectuels et la paysannerie roumaine de la fin du $\mathrm{xx}^{\mathrm{e}}$ siècle aux années 1930 », in Revue d'histoire moderne et contemporaine, $\mathrm{n}^{\circ} 1$, p. 144-155.

Durandin, C., 1994, L'engagement des intellectuels à l'Est. Memoires et analyses de Roumanie et de Hongrie, Paris, L'Harmattan.

Iorga, N., 1922, Formes byzantines et réalités balkaniques, Paris, Honoré Champion. Iorga, N., 1923, Relations entre Orient et l'Occident, Paris, J. Gamber Editeur.

Iorga, N., 1935, Byzance après Byzance. Continuation de l'histoire de la vie byzantine, Bucarest, Editions de l'Institut des Études byzantines.

Manolescu, N., 1976, Sadoveanu sau utopia cărții, București, Editura Eminescu.

Manolescu, N., 2008, Istoria critică a literaturii române. 5 secole de literatură, Pitești, Editura Paralela 45, p. 571-598.

Moura, J.-M., 1992, Lire l'exotisme, Paris, Dunod.

Ornea, Z., 1971, Sămănătorismul, București, Editura Minerva.

Sadoveanu, M., 1957, Zodia Cancerului sau vremea Ducăi-vodă, București, Editura de Stat pentru Literatură și artă. Trad. fr. Aurel George Boeșteanu, Le signe du Cancer, București, Editura Minerva, 1981. 
Simion, E., 2001, "Le double comme modele existentiel et culturel» in Figures $d u$ double dans les littératures européennes, ed. Gerard Conio, Cahiers du Cercel, Lausanne, L'Age d'Homme, p. 268-274.

Spiridon, M., 2004, Les dilemmes de l'identité aux confins de l'Europe: Le cas roumain, Paris, L'Harmattan.

Spiridon, M., 1982, Sadoveanu. Divanul înțeleptului cu lumea, București, Editura Albatros. 